\title{
TIME-CORRELATED SINGLE-PHOTON COUNTING RANGE PROFILING OF MOVING OBJECTS
}

\author{
Julia Hedborg*, Per Jonsson, Markus Henriksson, Lars Sjöqvist \\ Dept. of Electro-optical Systems, Swedish Defence Research Agency, FOI, PO Box 1165, SE-581 11 \\ Linköping, Sweden. *Email: julia.hedborg@foi.se
}

\begin{abstract}
Time-correlated single-photon counting (TCSPC) is a laser radar technique that can provide range profiling with very high resolution. Range profiles of multiple surface objects and geometrical shapes are revealed using multiple laser pulses with very low pulse energy. The method relies on accurate time measurements between a laser pulse sync signal and the registration of a single-photon event of reflected photons from a target.

TCSPC is a statistic method that requires an acquisition time and therefore the range profile of a non-stationary object (target) may be corrupted. Here, we present results showing that it is possible to reconstruct the range profile of a moving target and calculate the velocity of the target.
\end{abstract}

\section{INTRODUCTION}

The TCSPC technique offers the possibility to measure range profiles of stationary targets with resolution in the order of centimeters [1]. These profiles can be used for recognition and classification of remote optical systems or other reflecting objects.

The principle of TCSPC range profiling is based on time-of-flight measurements between the emitted laser pulse and the detection of a single photon. The registered photons from detection events are sorted into time bins according to their arrival time relative to the latest emitted laser pulse. These are typically presented as histograms with time bins on the $\mathrm{x}$-axis and counts on the $\mathrm{y}$ axis. The number of detected laser pulses should be less than $5 \%$ in order to avoid detector "pileup" effects and fulfil the Poisson statistic requirements [2]. This enables usage of very low laser power that may be an advantage for some applications and facilitates meeting eye-safety requirements.

A measurement of a stationary target, like a mirror or an optical system, will give a characteristic range profile (or signature), where multiple closely spaced surfaces are resolved.
However, if the target is moving, the profile is blurred over the time bins. Since the resolution is very high, a small movement is enough to corrupt the profile.

The aim of this work was to investigate the possibility of measuring range profiles of moving targets with a TCSPC system. By using the time information provided by the timing acquisition hardware we can reconstruct the characteristic range profile and also measure the velocity of the moving target.

\section{SYSTEM DESCRIPTION}

The main components of the experimental system are a picosecond pulsed laser, a transceiver unit for emittance of laser pulses and collection of reflected photons, a single-photon silicon detector and the timing acquisition hardware (Figure 1).

The laser is a super continuum (SC) fiber laser (Fianium Ltd. UK) emitting radiation from 450 to $1900 \mathrm{~nm}$. It operates at $40 \mathrm{MHz}$ pulse repetition frequency (or $25 \mathrm{~ns}$ pulse intervals) and has a pulse duration of 4 ps. An acousto-optical (AO) filter is used to select the wavelength and reduce the emitted laser power. A fixed wavelength of $832 \pm 5 \mathrm{~nm}$ was used in this work.

The detector is a SPAD (Single Photon Avalanche Diode) detector from Micro Photon Devices S.r.l. Italy, with a $100 \mu \mathrm{m}$ pixel diameter, $<250$ dark counts/s, 87 ns detector dead time and $<50 \mathrm{ps}$ timing jitter. A band pass filter $\left(\lambda_{c}=834 \mathrm{~nm}, \Delta \lambda=3 \mathrm{~nm}\right)$ is mounted in front of the detector to reduce the background radiation.

The timing acquisition hardware (PicoHarp300 from PicoQuant GmbH, Germany) registers the time between every photon detected by the SPAD device and the last electrical synchronisation pulse from the SC fiber laser.

A transceiver module with a wide field of view (FOV) adapted for short range laboratory measurements was used. The module is monostatic with the same optics for transmitting and collecting photons having a combined FOV of 
approximately $5 \mathrm{mrad}$. A CCD camera mounted next to the transceiver helped in aiming the system on the target.

The laser pulse frequency of $40 \mathrm{MHz}$, gives a range interval of $7.5 \mathrm{~m}$ between pulses and an ambiguity distance of $3.75 \mathrm{~m}$ for the system. The absolute distance to an object can be determined e.g. by using pseudo-random modulation of the laser pulse train $[3,4]$, but that feature is not implemented in the current set-up.

A more thorough description of the system is found in [5], except for the new monostatic wide FOV transceiver head.

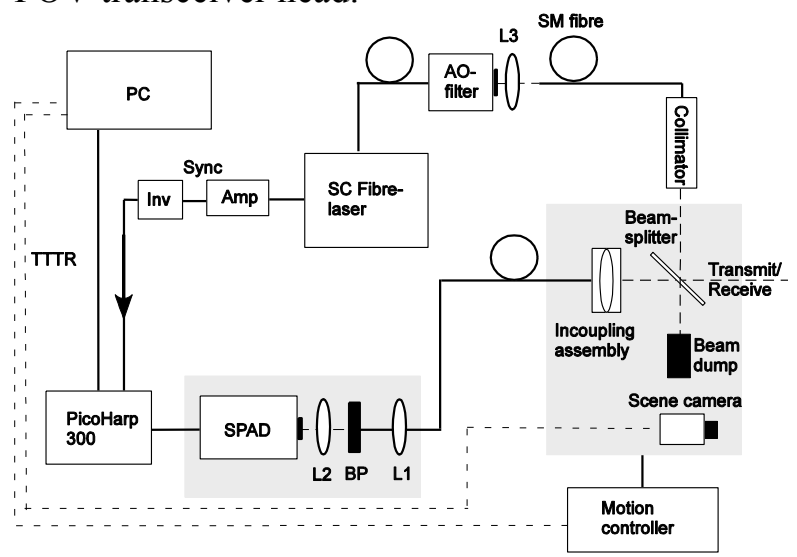

Figure 1. Overview of the TCSPS system used for the experiments. The transceiver module was mounted on a turn table. Abbreviations: SM (single mode), Amp (amplifier), Inv (inverter), TTTR (time-tagged time-resolved signal), Sync (laser pulse sync signal), L1 (collimating lens), L2/L3 (focusing lenses).

\section{EXPERIMENTAL METHOD}

For the experiments an indoor measurement facility was used. At a distance of about $50 \mathrm{~m}$ from the TCSPC transceiver, two different rails were positioned on which the targets were placed.

The first rail is $50 \mathrm{~cm}$ long with a motor driving step with constant velocity at the speed of a few $\mathrm{cm} / \mathrm{s}$. The second rail is $5 \mathrm{~m}$ long (longer than the ambiguity distance of $3.75 \mathrm{~m}$ ) and the targets were moved by pulling a line tied to a wagon comprising the mounted targets. The velocity was not constant and the rail not completely straight giving a slightly wobbly movement of the target.

The targets used for measurements were corner cube retro reflectors (CCRR) that provide strong reflections and a diffuse cone that gives a much weaker signal. First, static measurements were performed to provide reference range profiles. Subsequently measurements during movement of the targets on the two different rails were carried out.

The PicoHarp software was used to collect experimental data and in-house developed MATLAB routines were used off-line to analyse the results. The length of each time bin was set to 8 ps.

By using the PicoHarp TTTR (time-tagged time-resolved signal) mode for data recording both the time relative to the latest sync pulse and the number of sync pulses from the start of the measurement are recorded for every detected photon. The scheme enables reconstruction of the range profile of the moving object. This was done by sorting the signals into time intervals much shorter than the total acquisition time, and creating a histogram for every time interval (Figure 2).

By cross correlating the histogram from each time interval with the first one, the number of time bins the target has moved compared to the first time interval was calculated. This gives the distance as a function of time and by fitting a polynomic function to these values, a distance correction of every single detection event is calculated.

Though the number of counts for every time interval is low and the histograms noisy, a range profile that is very similar to a static measurement profile can be achieved. The reconstruction of the time intervals shown in Figure 2 is presented in Figure 3.

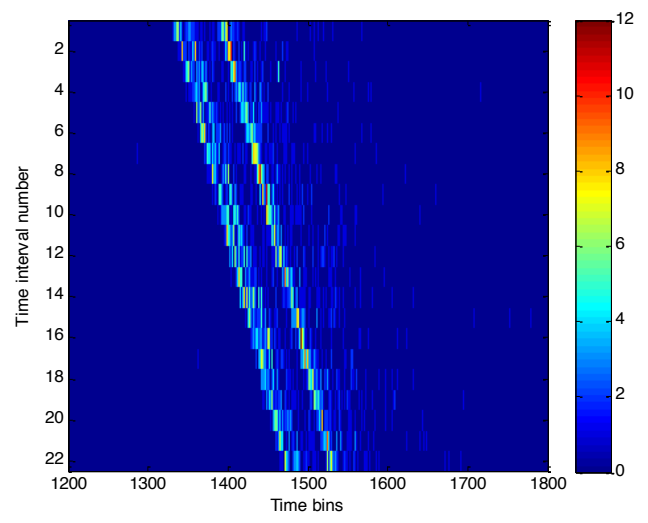

Figure 2. Histograms of every time interval (y-axis) plotted as a picture. The color scale represents number of counts for every time bin ( $x$-axis). The diagonal shape is a result of the movement of the object, in this case three CCRRs. 


\section{RESULTS}

To illustrate the reconstruction and the resolution of the system three different CCRRs were placed close to each other and moved on the short rail at a velocity of $0.025 \mathrm{~m} / \mathrm{s}$. CCRRs give strong reflections, but the signals were filtered to resemble longer distance or higher speed than was used. This results in few counts/s and it is not possible to achieve a good range profile by shortening the acquisition time because of the low count rate or by averaging over longer time intervals because of the movement. After the reconstruction, the range profile appears and is almost identical to a static measurement of the profile. This is illustrated in Figure 3 where one of the time interval histograms from Figure 2 is plotted together with the reconstructed and a static profile. The distance between the first two peaks (closest two CCRRs) of the range profile is $1.7 \mathrm{~cm}$.

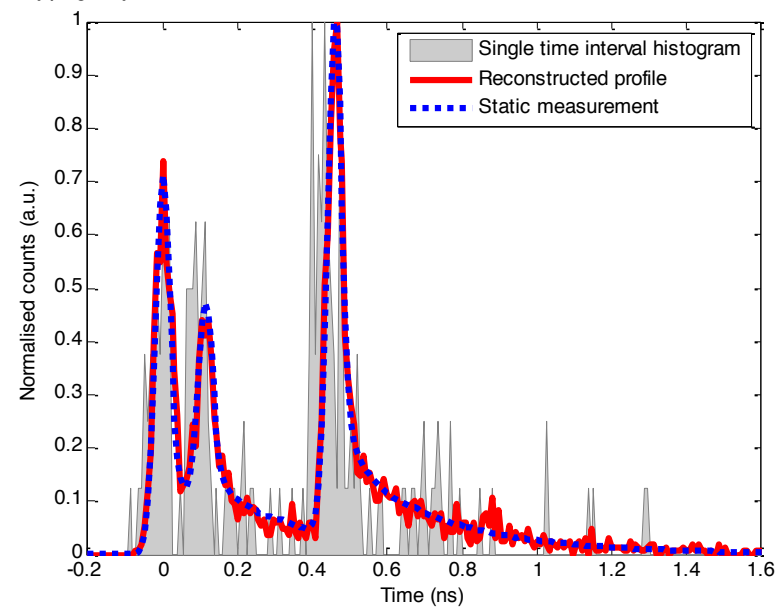

Figure 3. Three corner cubes closely placed on the short rail measured during movement. The gray area is one of the time interval histograms from Figure 2.

A static measurement of a single CCRR determines the system response and its FWHM (full width at half maximum), that is a measure of the system resolution, was 49 ps. This corresponds well to the specification of the detector, which gives the largest time jitter of the system components. For a more thorough characterisation of the system response, see [5].

The CCRR was subsequently mounted on the short and long rail, respectively, and studied during movement. The range profiles after reconstruction compared with the static profile are shown in Figure 4. The non-compensated histogram from this measurement before the reconstruction is also shown illustrating the broadening from $0.2 \mathrm{~m}$ movement during the measurement.

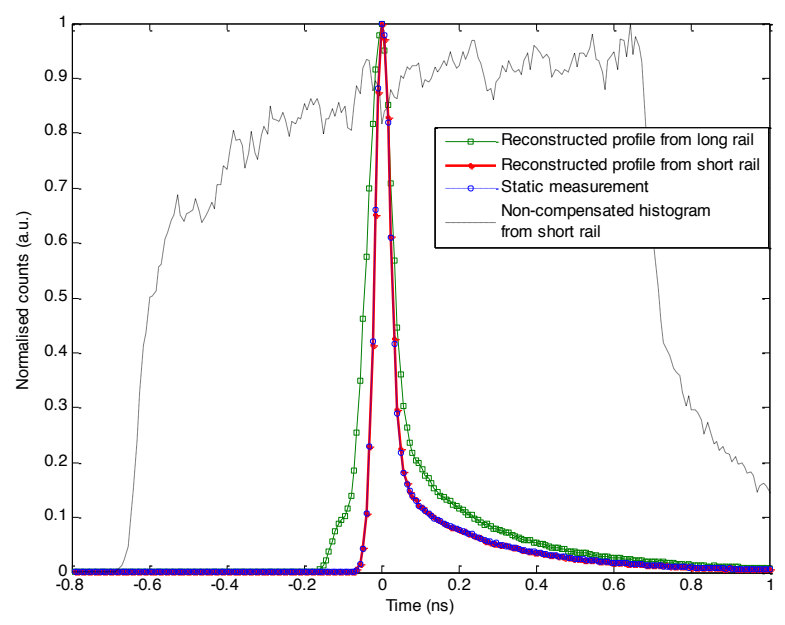

Figure 4. Range profile of the CCRRs, from static measurement and reconstructed range profiles from the measurement during movement on the long and short rails. The dotted line is the histogram of a moving CCRR as it looks before reconstruction.

The reconstructed range profile of the CCRR measured during movement on the short rail has the same FWHM as the static measurement. The velocity was calculated to 0.025 and $0.050 \mathrm{~m} / \mathrm{s}$ for two measurements at different speed settings. The reconstruction works excellent for both cases. Because of the strong signal a sampling time less than $0.05 \mathrm{~s}$ is required to achieve a characteristic range profile studying the CCRR.

On the longer rail, the movement was not controlled and nonlinear. However, the signals were successfully reconstructed for the CCRR. The FWHM was 81 ps which is broader than the static measurement, but the range profile is still recognisable (see Figure 4). The broadening is due to the non-linear movement that was not included in the fitted curve used for the reconstruction. The difference of fitted curve and the real velocity is illustrated in Figure 5. The average velocity for this measurement was $0.32 \mathrm{~m} / \mathrm{s}$.

The studied cone was $30 \mathrm{~cm}$ high and had a base diameter of $30 \mathrm{~cm}$ with a white diffuse surface. Since the reflection of the cone is weak compared to the CCRR, longer time intervals $(0.2-0.3 \mathrm{~s})$ was required for the reconstruction in 


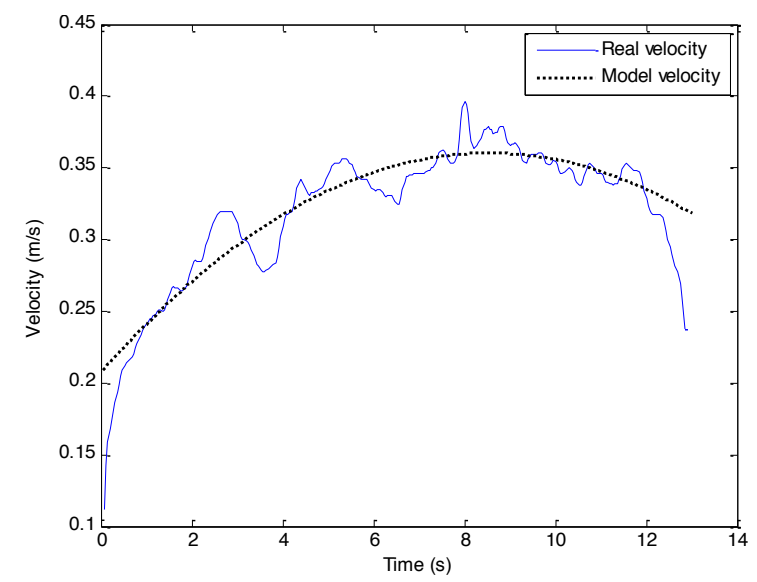

Figure 5. Speed as a function of time for the corner cube traveling on the long rail. The dotted line represents the model curve used for the reconstruction.

order to obtain histograms useable for the crosscorrelation. Figure 6 shows the cone profile from the static measurement and the reconstructed profile from the measurement during movement on the short rail.

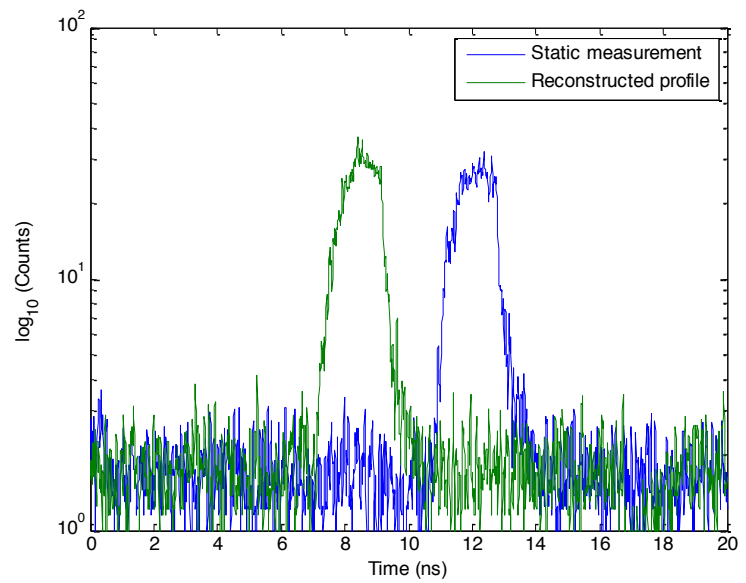

Figure 6. Range profiles for the cone from the static measurement (blue) and the reconstructed range profile from measurement during movement (green). The curves were filtered with a gaussian filter to reduce some noise.

There is a slight difference between the two profiles, which are time shifted for better visualisation. The FWHM of the static cone profile was $1.47 \mathrm{~ns}$ and about the same for the reconstructed signal. The average velocity was calculated to $0.027 \mathrm{~m} / \mathrm{s}$, a little higher than the speed calculated from the cube measurement which should be more correct. A measurement at $0.05 \mathrm{~m} / \mathrm{s}$ was also performed with similar result.

\section{CONCLUSIONS}

The TCSPC system enables high-resolution characterisation of remote, retro reflecting or reflecting objects using low power eye-safe laser illumination. The presented results show that it is possible to reconstruct range profiles of moving object with this technique. This technique works even when the necessary integration time is longer than the period for which the movement is negligible, i.e. for objects with low signal strength compared to the velocity. Reconstruction of the signal requires no prior information of the original range profile and the instantaneous and average velocity of the object can be calculated. Objects moving longer distances than the ambiguity distances can also be compensated with respect to velocity induced broadening.

With further developed reconstruction algorithms, objects moving at greater velocity or in more nonlinear paths should be possible to identify or classify.

\section{ACKNOWLEDGEMENT}

This work has been supported by the Swedish Armed Forces research program and the Swedish Defence Materiel Administration (FMV).

\section{REFERENCES}

[1] G. Buller, A. Wallace, 2007: Ranging and Three-Dimensional Imaging Using TimeCorrelated Single-Photon Counting and Point-byPoint Acquisition, IEEE J. Sel. Top. in Quant. Electr.,13(4), 1006-1015.

[2] G. Buller, R. Collins, 2010: Single-photon generation and detection, Meas. Sci. Technol, 21(1), 012002.

[3] N. J. Krichel, A. McCarthy, G. S. Buller, 2010: Resolving range ambiguity in a photon counting depth imager operating at kilometer distances, Opt. Express, 18(9), 9192-9206.

[4] P. Hiskett, C. S. Parry, A. McCarthy, G. S. Buller, 2008: A photon-counting time-of-flight ranging technique developed for the avoidance of range ambiguity at gigahertz clock rates, Opt. Express, 16(18), 13685-13698.

[5] L. Sjöqvist, M. Henriksson, P. Jonsson, O. Steinvall, 2014: Time-correlated single-photon counting range profiling and reflectance tomographic imaging, Adv. Opt. Techn., 3(2), 187-197. 